\title{
Análisis de recursos turísticos para el cálculo del índice de atractividad. Caso de estudio Loma de la cruz, Holguín
}

DOI: https://doi.org/10.33262/ap.v3i3.2.97

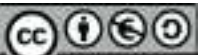

Analysis of tourism resources for the calculation of the attractiveness index. Case study Loma de la Cruz, Holguin

Solangel Pérez-Labrada. ${ }^{1}$, Félix Díaz-Pompa. ${ }^{2}$, Beatriz Serrano-Leyva. ${ }^{3}$ \& Nolberto Cruz-Aguilera. ${ }^{4}$

\begin{abstract}
.
Tourist resources and their attractions constitute the fundamental basis of tourism, and it is essential to analyze and evaluate them in order to determine the tourist potential of an area and, consequently, its viability as a support for the development of this activity. The present work was intended to analyze the attractiveness of Loma de la Cruz, taking into account its geographic and patrimonial values. Theoretical methods were used for the analysis and determination of the background, characterization, conceptualization of the object and for the processing of information, as well as empirical methods for the review and analysis of documentary and non-documentary sources, information and communication technologies. The Loma de la Cruz is a natural viewpoint of the city full of traditions, culture and identity that encourages any visitor to climb it in a mandatory

\footnotetext{
${ }^{1}$ Universidad de Holguín, Facultad de Ciencias Empresariales y Administración, Carrera Licenciatura en Turismo, Holguín, Cuba, solangelperezlabrada@gmail.com

${ }^{2}$ Universidad de Holguín, Facultad de Ciencias Empresariales y Administración, Carrera Licenciatura en Turismo, Holguín, Cuba. felixdp1978@gmail.com

${ }^{3}$ Universidad de Holguín, Facultad de Ciencias Empresariales y Administración, Carrera Licenciatura en Turismo, Holguín, Cuba. bettys11997@gmail.com

${ }^{4}$ Universidad de Holguín, Facultad de Ciencias Empresariales y Administración, Carrera Licenciatura en Turismo, Holguín, Cuba. nolbert1309@gmail.com
} 
way as a record of its passage through the city, which has restaurants that provide services to those who visit it, forming a perfect complement as a natural attraction. The development of tourism in the attraction has had a negative impact on the conservation of both natural and heritage elements. The heritage values associated with the property include artistic and cultural, historical, social, economic, functional, natural and environmental values. It is a natural site that contains historical and ethnographic values that over the years have symbolically represented the city of Holguín. According to the quantitative and qualitative evaluation of the Attractiveness Index, it is classified as an attractive place with a high attractiveness index; however, there are indicators with low indexes such as lodging capacity; tourist organization, integration and management; transportation, access and roads; and level of conservation and environmental conditions.

Keywords: tourism resources, attractions, attractiveness index, heritage, geography

\section{Resumen.}

Los recursos turísticos y sus atractivos constituyen el cimiento principal del turismo, siendo ineludible su análisis y evaluación, para precisar las potencialidades turísticas de una zona y, como resultado, su viabilidad como soporte para impulsar el desarrollo de esta actividad. El presente trabajo se enfocó en realizar un análisis del atractivo Loma de la Cruz teniendo en cuenta los valores geográficos y patrimoniales del mismo. Se utilizaron métodos teóricos para el análisis y determinación de los antecedentes, caracterización, conceptualización del objeto y para el procesamiento de la información, así como métodos empíricos para la revisión y análisis de fuentes documentales y no documentales, tecnologías de información y comunicaciones. La Loma de la Cruz es un mirador natural de la ciudad colmado de tradiciones, cultura e identidad que incita a cualquier visitante a escalarla de modo obligatorio como constancia de su paso por la ciudad, que al poseer restaurantes que brindan servicios a los que la visitan, forman un perfecto complemento como atractivo natural. El desarrollo del turismo en el atractivo ha incidido negativamente en la conservación de elementos tanto naturales como patrimoniales. Dentro de los valores patrimoniales asociados al bien están los valores artístico y cultural, histórico, social, económico, funcional y natural y ambiental. Es un sitio natural que encierra valores históricos y etnográficos que han hecho a través de los años que haya representado simbólicamente a la ciudad de Holguín. De acuerdo a la evaluación cuantitativa y cualitativa del Índice de Atractividad se clasifica como un lugar atractivo y con un alto índice de atractividad, sin embargo, existen indicadores con bajos índices como capacidad de alojamiento; organización, integración y gestión turística; transporte, acceso y vialidad; y nivel de conservación y condiciones del entorno.

Palabras claves: recursos turísticos, atractivos, índice de atractividad, patrimonio, geografía. 


\section{Introducción.}

El turismo es el sector de mayor crecimiento que articula un conjunto entramado de relaciones económicas y ejerce una poderosa influencia en la dinámica de numerosas regiones y países (Ortiz-Liñán \& Vázquez-Solís, 2021). Además del crecimiento del Producto Interno Bruto que incentiva, observable en el número de empleos e ingresos directos e indirectos, en 2019, el valor de las exportaciones asociadas al turismo fue de 1.7 billones de dólares y el gasto superó al crecimiento económico mundial (OMT, 2019). Provocando que los distintos gobiernos y entidades privadas le otorguen una mayor atención promoviendo diferentes programas y actividades de fomento a la actividad turística (Gambarota \& Lorda, 2018).

(Aranibar, 2021) hace referencia al turismo, en una concepción amplia de entendimiento, como una "Actividad económica, social, medioambiental y humana", que involucra varios elementos y aspectos, que en términos de planificación y gestión espacial, se constituyen en recursos turísticos, recursos que pueden ser de oferta o demanda.

Los atractivos y recursos turísticos son considerados como la base del desarrollo turístico de una localidad, debido a que el turismo es esencial para el desarrollo de una localidad, es importante invertir en ellos para fortalecer el patrimonio, la identidad cultural, conservarlos y de igual forma motivar a los turistas para que realicen una visita.

En tal sentido, La Organización Mundial del Turismo (OMT) hace referencia de que un recurso turístico son todos los bienes y servicios presentes en un determinado ámbito geográfico, capaces de atraer por sí mismo visitantes, hacen viable el desarrollo de la actividad turística y satisfacen las necesidades de la demanda, mediante la actividad del hombre y de los medios que cuenta (Carvajal-Zambrano \& Lemoine-Quintero, 2018). Mientras que, los atractivos turísticos son todos aquellos elementos materiales y/o inmateriales que son aptos de ser transformados en un producto turístico con potencialidad para influir sobre el proceso de decisión del turista y como resultado, provoca su visita a través de las distintas vías de desplazamiento desde el lugar de residencia habitual hacia un territorio turístico (Rivera, 2008).

Asociado a la actividad turística y a sus recursos y atractivos está el patrimonio, como herencia cultural propia de una comunidad, región o nación que se transmite de generación en generación (Díaz, 2013), y que es definido como el conjunto de bienes culturales y naturales con los que cuenta un territorio que constituyen la identidad del lugar y de sus habitantes (UNESCO, 2017). Este patrimonio de orden territorial vincula tanto los recursos naturales como los culturales que se han heredado en una zona geográfica y que además cuentan con una notable aprobación y aprecio social (CantúMartínez, 2018).

Los recursos naturales, deportivos y culturales, religiosos, eventos, características del clima, y gastronomía entre otros son los factores de atractividad de un destino turístico (Beerli \& Martin, 2004; Mondéjar \& Vargas, 2009). En tal sentido, para (Lee, Ou, \& Huang, 2009; Wu, Xie, \& Tsai, 2015) la atractividad turística es definida como un paquete 
de equipamientos y servicios los cuales están compuestos, además, por atributos multidimensionales. Desde el punto de vista de los clientes, entidades y la competencia en general de un destino turístico, (Beirman, 2003) argumenta que es la capacidad que posee un lugar de interés de atraer nuevos clientes, al cual se le añade generalmente un valor cultural inherente o exhibido, que resalta por su significado histórico, por su belleza natural o artificial.

En este contexto, según (Magallanes, 2004) citado por (Medina-Labrada, Pérez-Ricardo, \& Riquenes-Gainza, 2019), constituye un aspecto fundamental el análisis de la atractividad de destinos turísticos, pues constituye una herramienta esencial para identificar negocios turísticos que partir de su evaluación se consideran más atractivos y con mejores condiciones para insertarse en la competencia, además, contribuye a dar solución a un problema cardinal en la incorrecta gestión de los negocios turísticos.

Uno de los lugares emblemáticos, lleno de tradiciones que existen en la ciudad de Holguín, es la Loma de la Cruz, sitio de obligatoria visita para todo viajero al arribar a la ciudad (Guzmán-Vilar \& García-Vidal, 2014). Declarada Monumento Histórico Arqueológico Colonial de la isla de Cuba, con una altura de 276,9m y 458 escalones, descansos intermedios y bancos incluidos que a mitad del ascenso pueden parecer los más cómodos del mundo, la réplica de lo que fue originalmente un fuerte militar, una pequeña capilla y una plazoleta con un podio, que se utiliza usualmente como altar, sobre el cual se erige, majestuosa, una enorme cruz de madera, constituye un símbolo de la ciudad holguinera. Es un sitio natural que encierra valores históricos y etnográficos que han hecho a través de los años que haya representado simbólicamente a la ciudad de Holguín, no obstante, el desarrollo del turismo en el atractivo ha incidido negativamente en la conservación de elementos tanto naturales como patrimoniales.

\section{Metodología.}

En este trabajo se realiza un análisis del atractivo Loma de la Cruz teniendo en cuenta los valores geográficos y patrimoniales del mismo. Para el mismo se hace la revisión y análisis de fuentes documentales y no documentales, tecnologías de información y comunicaciones.

El procedimiento para para hallar el índice de atractividad de la "Loma de la Cruz" incluyó:

a) Análisis de los aspectos físico-geográficos, geoturísticos y diagnóstico ambiental

b) Análisis de los recursos patrimoniales

c) Confección de la Matriz de Índice de Atractividad

La matriz está compuesta por treinta atributos o indicadores a evaluar en cuanto a importancia, dándole valores de 1 a 3 de acuerdo a su nivel de importancia, y a la posición relativa, que se evalúa dando valores de $-10,-5,-2,2,5,10$ de acuerdo al comportamiento o estado actual del atributo. Para hallar el Valor del atributo, se multiplica el valor de la 
Importancia por el de la Posición relativa, repitiendo esta operación para cada atributo de la tabla. Por último, se calcula el Índice de Atractividad mediante la sumatoria de todos los valores de los atributos y dividiendo el total entre la cantidad de atributos (Tabla 1 y Tabla 2)

Tabla 1. Fórmulas para calcular el Índice de Atractividad y el Valor del Atributo

\begin{tabular}{llll}
\hline \multicolumn{2}{c}{ Cálculo del Índice de Atractividad } & \multicolumn{2}{c}{ Cálculo del Valor del Atributo } \\
\hline Fórmula & $\mathrm{Ia}=\sum(\mathrm{Va} 1+\mathrm{Va} 2+\ldots+\mathrm{Va} 30)$ & Fórmula & $\mathrm{Va}=\mathrm{Im} \mathrm{x} \operatorname{Pr}$ \\
& $/ \mathrm{N}$ & & \\
\hline \multirow{3}{*}{ Donde } & $\mathrm{Ia}-$ Índice de atractividad & & $\mathrm{Va}-$ Valor del atributo \\
& $\mathrm{Va}-$ Valor del atributo & Donde & $\mathrm{Im}-$ Importancia \\
& $\mathrm{N}-$ Número de atributos & & $\mathrm{Pr}-$ Posición relativa \\
\hline
\end{tabular}

Fuente: Elaboración propia.

Tabla 2. Evaluación cuantitativa y cualitativa del Índice de Atractividad

\begin{tabular}{cc}
\hline \multicolumn{2}{c}{ Evaluación cuantitativa y cualitativa del Índice de Atractividad (Ia) } \\
\hline $\begin{array}{c}\text { Valores del Índice de } \\
\text { Atractividad (Ia) }\end{array}$ & $\begin{array}{c}\text { Evaluación Cualitativa del Índice de } \\
\text { Atractividad (Ia) }\end{array}$ \\
\hline$-20,8$ a $-30,0$ & Muy poco atractivo \\
$-11,4$ a $-20,7$ & Medianamente poco atractivo \\
$-2,0$ a $-11,3$ & Poco atractivo \\
2,0 a 11,3 & Atractivo \\
11,4 a 20,7 & Medianamente atractivo \\
20,8 a 30,0 & Muy atractivo \\
\hline
\end{tabular}

Fuente: Elaboración propia.

\section{Resultados.}

A continuación, se realiza el diagnóstico del atractivo turístico "Loma de la Cruz" de la ciudad de Holguín.

\section{Análisis de los aspectos físico-geográficos, geoturísticos y diagnóstico ambiental}

\section{Localización geográfica:}

En las alturas de Maniabón, al noroeste de la ciudad de Holguín, en los 2053'58’ de latitud Norte y los $76^{\circ} 16^{\prime} 08^{\prime \prime}$ de longitud Oeste, municipio de Holguín. Altura de 276,9 m, elevada unos 130 metros con relación al valle donde se asienta Holguín. Se puede divisar a gran distancia, es punto natural de referencia para localizar la ciudad de Holguín.

Tipo de entorno turístico: natural - urbano.

\section{Características del medio físico-geográfico:}

Mole serpentinítica de 276,9 m de altura, se eleva al norte de la ciudad a unos 2,5 km de la iglesia de San Isidoro. Perteneciente al grupo orográfico Maniabón, cuya silueta es visible desde larga distancia, sirve de punto de referencia para localizar la urbe. 
La geología se caracteriza por encontrarse enclavada en el seno de un complejo de rocas magmáticas pertenecientes al cinturón ofiolítico del jurásico medio hasta el superior, constituida por rocas del cretácico superior, como serpentinitas, peridotitas y dunitas. (González, Oca, \& González, 2010)

El clima se caracteriza por temperatura media entre $18{ }^{\circ} \mathrm{C}$ y $22{ }^{\circ} \mathrm{C}$, y los acumulados anuales de precipitación superan $1200 \mathrm{~mm}$. El viento se distingue por sus características orográficas, si se tiene en cuenta la fase de sequía, la cual es muy típica en esta provincia durante los últimos años, su dirección es del $\mathrm{NE}$ con 10,8 a 12,6 Km/h de velocidad promedio. (González et al., 2010)

Su suelo es del tipo nipe-ferrítico púrpura, poco ondulado, erosionado, bien drenado y pobre desde el punto de vista agrícola. (González et al., 2010)

Presenta una vegetación xeromorfa espinosa, conformada por arbustos y especies introducidas, y en algunas zonas encontramos que la vegetación es de cuabales, predominan plantas espinosas, con hojas pequeñas y duras, muy resistentes a la sequía, algunas endémicas de la provincia como el cactus enano de Holguín, Melocactus de Holguín, Roble de sabana, Yuraguana de Holguín y la Rosa de Sabana, considerada un símbolo de la provincia. (González et al., 2010)

La fauna dentro del área es bastante diversa en cuanto a grupos zoológicos (Annelida, Nematoda, Diplopoda, Chilopoda, Crustacea, Insecta, Arachida, Mollusca, Amphibia, Reptilia, Aves, Mammalia) al igual que en cualquier otra parte del territorio holguinero. (Cubaism, 2018; Igarza et al., 2008), lo cual se ejemplifica en la Tabla 3.

Tabla 3. Fauna existente en La Loma de la Cruz

\begin{tabular}{ll}
\hline Grupo taxonómicos & \multicolumn{1}{c}{ FAUNA } \\
& $\begin{array}{l}\text { Mariposas diurnas: Eurema nicipe, Heliconius charitonius, } \\
\text { Drya julia y Holguinia holguin }\end{array}$ \\
\hline Arácnidos & $\begin{array}{l}\text { Araña peluda (endemismo cubano) } \\
\text { Escorpiones: Rhopalurus junceus (alacrán colorado), } \\
\text { Centruroides baracoe; y Microtityus fundorai fundorai } \\
\text { (endemismo cubano) }\end{array}$ \\
\hline Zoluscos & Zachrysia gundlachiana (endemismo oriental) \\
\hline Anfibios & Osteopilus septemtrionalis (rana platanera) \\
& Eleutherodactylus atkinsis (ranita de muslos rojos) \\
\hline Reptiles & Anolisallisoni (caguayoverde-azul) \\
& Anolissagrei (caguayo o torito) \\
\hline Aves & Mimus polyglottos (Sinsonte) \\
& Dives atroviolaceus (Totí, endemismo cubano) \\
& Chiscalus niger (Chinchiguaco) \\
& Crotophaga ani (Judío) \\
& Columbina passerina (Tojosa) \\
& Dendroicatigrina \\
& Sethophaga ruticila \\
& Passer domesticus (gorriones) \\
\hline
\end{tabular}


Tabla 3. Fauna existente en La Loma de la Cruz (continuación)

\section{FAUNA}

\begin{tabular}{ll}
\hline Mamíferos & Molossus molossus (murciélago casero) \\
& Artibeus jamaicensis (murciélago frutero) \\
\hline Roedores & Mus musculus (guayabito) \\
& Rattus rattus (rata negra) \\
& Rattus norvergicus (rata parda) \\
\hline
\end{tabular}

Fuente: Elaboración propia.

\section{Estructura territorial pre-turística:}

El poblamiento inicial no pasó más allá de una finca con un grupo de españoles, indios y algún africano traído como esclavo. Si en aquella época crecían algunos árboles en sus laderas los vecinos del hato se encargaron de talarlos para sus construcciones y fogones. (Obregón, 1995)

Desde la etapa hispánica resultó el lugar más significativo relacionado con la tradición religiosa de las Romerías de la Cruz, así como destino de peregrinaciones; se consideró como punto estratégico para formar parte del sistema defensivo de la ciudad e incluso se colocó un heliógrafo que proporcionaba la comunicación con el norte de la jurisdicción y el pueblo de Jiguaní; además de tener una participación activa en el trazado original del esquema urbano, pues a partir de esta se delinearon las plazas, manzanas y calles, las cuales debían ser lo más rectas y regulares posibles en correspondencia con lo regulado por las Leyes de Indias; asimismo su protagonismo se hizo presente cuando se rectificó el primitivo trazado al ser otorgado el título de ciudad.

A partir de 1956, se emplea en la mejora de las comunicaciones con la instalación de una planta transmisora y receptora de radio y televisión. (Cepero, Hidalgo, Ramírez, Cruz, \& Beltrán, 2016)

\section{Variables territoriales:}

En la actualidad se considera el mirador natural de la ciudad y un lugar significativo dentro de la celebración de las Romerías de Mayo, evento de gran connotación nacional e internacional.

En su cima hay varias instalaciones, entre las que se destacan: la estación repetidora de señales de televisión, mirador, restaurante y plazoleta-mirador con torre de observación.

En las faldas se edificaron gran número de viviendas, pese a la prohibición del ayuntamiento colonial de la construcción al menos $3 \mathrm{~km}$ alrededor. La ladera sur se encuentra desprovista de estas, por la empinada de esta vertiente.

\section{Recursos turísticos naturales:}

Es un mirador natural de la ciudad colmado de tradiciones, cultura e identidad que incita a cualquier visitante a escalarla de modo obligatorio como constancia de su paso por la 
ciudad, que al poseer restaurantes que brindan servicios a los que la visitan, forman un perfecto complemento como atractivo natural.

El relieve constituye otro recurso turístico natural de este sitio, pues la elección de un destino de montaña depende, mucho más que en el caso de los litorales, de las verdaderas características topográficas del macizo.

Otro recurso es el paisaje, un sistema espacial objetivamente existente, homogéneo en sus diferentes partes, formado bajo la influencia de los procesos naturales o de la actividad modificadora de la sociedad. En la Loma de la Cruz el paisaje como recurso turístico ha experimentado transformaciones constantes, debido a una acción inadecuada del hombre y su consecuente degradación.

\section{Carácter enclavado o integrado del espacio turístico en la estructura territorial:}

El destino turístico Holguín se define por su integralidad y variedad, donde se combinan de forma singular el sol, la playa, la naturaleza, la historia, la arqueología, la cultura y las tradiciones locales; lo que conlleva a que sea un destino preferido por su autenticidad y diversidad. La Loma de la Cruz, símbolo de la ciudad, se integra perfectamente al entorno holguinero, constituyendo el mirador natural por excelencia de la majestuosa Ciudad de los Parques.

\section{Problemas ambientales y consecuencias del desarrollo turístico:}

En lo que respecta a la Loma de la Cruz el turismo ha incidido negativamente en la conservación de elementos tanto naturales como patrimoniales, pues al incrementarse el número de visitas al sitio, también se produce un incremento de la acción del hombre sobre el espacio geográfico sobre el cual se localiza este producto turístico, pudiéndose ver modificado el hábitat de muchas especies de la flora y la fauna de este territorio.

Resultados de las exploraciones realizadas en octubre de 2004 por un equipo del Centro de Investigaciones y Servicios Ambientales y Tecnológicos (CISAT-CITMA), evidenciaron que la cantidad de especies registradas fue baja, lo cual es una consecuencia de la intensa modificación de los hábitats originales, debido a la tala de la vegetación, incendios periódicos que han afectado el área y a la invasión de especies de plantas exóticas, lo que ha transformado a los cuabales en sabanas antrópicas, y por ende ha provocado una reducción de la riqueza de especies.

\section{Análisis de los recursos patrimoniales:}

Denominación: Con el nombre de cerro Bayado fue conocida inicialmente esa pequeña elevación de 261 metros sobre el nivel del mar, hasta que adoptó su actual nombre a partir del 3 de mayo de 1790, cuando el fraile de la comunidad franciscana Antonio de Alegría llegó hasta su cima y colocó allí una cruz de madera bendecida, presuntamente para evitar epidemias y catástrofes. (Isla Local, 2017) 
Clasificación: Este sitio se considera mixto pues posee diferentes valores patrimoniales, tanto bienes naturales como culturales, así como material e inmaterial, en este último tanto mueble como inmueble.

Tipología: Patrimonio material y paisaje cultural en el cual coincide un conjunto histórico, integrándose la vegetación con un enclave arquitectónico de la época colonial, además un lugar ritual donde se alza la cruz de la iglesia católica.

Señalización: Desde lugares más céntricos de la ciudad se pueden observar señales que indican cómo llegar a la Loma de la Cruz, ya sea por la escalinata o por la carretera.

Declaratoria: Declarado hoy día Monumento Histórico Arqueológico Colonial de la isla de Cuba. ("Ciudad de Holguín - Ciudad de los Parques," 2018)

Identificación: No se ubica ninguna placa establecida por la Comisión Nacional de Monumento que identifica que el bien está declarado.

Propiedad: Es una propiedad estatal socialista.

\section{Valores patrimoniales asociados al bien:}

Valor artístico y cultural: El fuerte que tiene un diseño arquitectónico que responde a la fortaleza de la época colonial.

Es escenario del evento más importante de la provincia las Romerías de Mayo, el cual es promocionado por la Asociación Hermanos Saiz con el objetivo de promover el Arte Joven; además el espacio que comprende la rotonda, fuerte militar y la base del cerro se inserta a la vida cultural holguinera mediante el desarrollo in-situ de las más variadas expresiones artísticas locales como la música, la danza y las artes visuales.

Valor histórico: Al inicio de la guerra del 68, el cerro de la Cruz fue fortificado, primero con una torre de mampostería y posteriormente con un fortín de base cuadrada, pues desde época temprana su localización fue considerada un sitio estratégico que permitía la visibilidad desde una gran distancia.

Las faldas de la elevación también eran utilizadas por el Ejército Español, al servir de paredón de fusilamiento de los patriotas que eran apresados.

En 1895 el Regimiento de la Habana edifico un fuerte con base rectangular y aspilleras, en el cual fue colocado un heliógrafo que se comunicaba con otro que se había colocado en Jaguaní. (Obregón, 2010)

Valor social: el sitio representa expresiones inmateriales como las tradiciones de sus pobladores, en cuanto a las cuestiones religiosas que se sostiene emerge una antigua tradición católica revivida por el fraile franciscano Antonio de Alegrías, el cual ascendió a la cima del cerro el 3 de mayo de 1790 con la Cruz de Cristo a cuesta, y allí la situó para prevenir enfermedades, desastres naturales o malos presagios; lo cierto es que con este acontecimiento se inició una creencia calificada como los Días de la Cruz, la cual 
consistía en realizar una procesión acompañada de una banda de música que nacía de la Catedral de San Isidoro y terminaba en la loma donde se oficializaba la misa; asimismo esta costumbre se llevó a cabo los Domingos de Resurrección, entre las prácticas más usuales se hallaban regar flores, orar y pagar promesas, estas podían consistir en subir la loma en rodillas o descalzos.

En 1994, producto de la labor de promotores culturales locales se revive la tradición de las Romerías de Mayo, convertida en un evento que transciende a nivel nacional e internacional con la duración de siete días. Este suceso cultural logró revivir la tradición, aunque renovada, y sobre todo incentivar en las nuevas generaciones la pertenencia por el lugar.

La Loma de la Cruz no ha sido tan solo marco propicio para la festividad católica de la Romerías de la Cruz, también en ella se han manifestado otras creencias, propias del sincretismo religioso que caracteriza la cultura cubana, en las alturas habita un Obatalá, pero no es usual realizar este rito en Holguín. Se dice que la liturgia espiritual es la que más uso le da a esta elevación ya que allí se venera la Virgen de la Caridad, Patrona de Cuba; también a San Isidoro, Patrón de Holguín". Pero lo cierto es que en el altar de la cruz se mezclan toda una serie de mezclas y bilongos; velas encendidas diecisiete centavos para San Lázaro, "trabajos" con cintas rojas, manojo de yerbas, animales muertos, etc. Lo más común es que la mayoría de las personas cumplen promesas subiendo tan solo la loma. Unos descalzos, otros en cuatro pies, encienden velas cuando un santo devoto le concedió lo que pidió. (Pupo, Suárez, Pérez, \& Figueroa, 2012)

Uno de los acontecimientos más importantes en la actualidad asociados a la Loma de la Cruz fue la visita del Papa Francisco a la ciudad de Holguín, donde en horas de la tarde del lunes 21 de septiembre del 2015, el Sumo Pontífice bendijo a la ciudad desde uno de los símbolos más emblemáticos de la ciudad. En este bello paraje, se realiza una breve ceremonia, en la que se le ofrece al Sumo Pontífice detalles del emblemático lugar por parte de las autoridades eclesiásticas, finalmente, el Papa Francisco concluye agradeciendo y felicitando a todos los presentes y bendice a la hermosa Ciudad de los Parques. (Radio Rebelde, 2015)

Valor económico: desde 1928 las autoridades locales vieron la posibilidad de incluir la Loma de la Cruz como atractivo turístico de obligada visita para los forasteros. En la actualidad se cuenta con servicios gastronómicos; se han trazado proyectos para la construcción de instalaciones hoteleras; sin embargo, dada las potencialidades que posee y además por catalogarse como símbolo de la ciudad de Holguín, incluso existe la frase muy popularizada «Quien venga a Holguín y no suba la Loma de la Cruz, no ha venido a Holguín». (Obregón, 2015) No se gestiona de forma adecuada como recurso turístico.

Valor funcional: resulta el mirador natural de Holguín, pero se considera dado los valores auténticos que encierra, que se podrían reanimar otros atributos idóneos para dar vida al lugar, rescatar los bienes materiales e inmateriales que este concentra, mejorar el hábitat, 
y por ende hacerlo más participativo en la cotidianidad del holguinero y más placentero en el disfrute del visitante.

Valor natural - ambiental: Sitio natural que encierra valores históricos y etnográficos que representa simbólicamente la ciudad.

Estado de conservación: El estado de conservación es regular, pero en la actualidad se trabaja para conservar los bienes materiales que presenta.

Integridad: El sitio es transformado, dado en su localización apartada ha sido objeto de maltratos por parte de personas que no reconocen sus valores patrimoniales, así como al encontrarse al aire libre, está bajo la influencia de las inclemencias del tiempo al que ha estado sometido, tales como incendios y huracanes, por lo que ha sido necesario restaurar en varias ocasiones este sitio

\section{Descripción de los elementos tangibles e intangibles del bien:}

Se trata de un accidente geográfico ubicado al norte de Holguín a 261 metros sobre el nivel del mar, un sitio natural por excelencia que contiene sus particularidades geográficas en el suelo, la vegetación y la fauna. Tuvo gran influencia en la fundación de la ciudad y su posterior evolución, ha constituido en elemento esencial en el sistema defensivo de la ciudad, de ahí que en este se encuentren construcciones militares de la etapa hispánica. Se encuentra además su repercusión religiosa, lo que recrea las costumbres y creencias de un grupo social.

Contiene construcciones militares y civiles. En el siglo XIX, inicio de la Guerra del 68, el cerro se fortificó con una torre de mampostería llamada Numancia y luego se incluyó un fortín de base cuadrada con aspilleras, planta, piso y azotea realizado con materiales de ladrillos nombrado La Vigía; y en el siglo XX, en el año 1927 se inició un proyecto arquitectónico realizado por el ingeniero Vicente Biosca que incluía para mejorar el acceso a la construcción una escalinata con 458 escalones iniciada desde la base hasta la cima del cerro que incluye plataformas de descansos y bancos, una plazoleta o parque en forma de rotonda en la cima de la loma que contenía en su centro el fortín ya establecido siglo atrás y que resultó reconstruido, un oratorio que comprendía la cruz de madera, y dos miradores. La rotonda se concibió rodeada de bancos y con dos entradas que permitían el acceso al oratorio y a los miradores; hacia estos últimos se aprecian dos escalinatas de pequeñas dimensiones, una hacia el denominado Balcón de Gibara y la otra Balcón de Holguín, que aparece en la misma dirección donde se encuentra el oratorio con un podio que sirve de altar y en su interior un nicho donde se depositan las ofrendas y sobre el cual está colocada la cruz de madera. Tanto la rotonda como los miradores, se concibieron de estilo ecléctico; en el caso de la rotonda es circundada por un muro perimetral y ornamentada por pináculos y piñas de remate, mientras que los miradores son protegidos por balaustradas.

En este sitio se han desarrollado fiestas populares que han recreado comidas y bebidas tradicionales, y modos de vida de la población, además de leyendas populares y el 
sincretismo religioso de los pobladores; aspectos que, en su conjunto, así como la repercusión que ha tenido en todos los aspectos de la historia local, hace que este exponente sea una parte indisoluble en la formación de la identidad cultural del holguinero.

\section{Valoración de la difusión que se realiza del exponente como producto cultural:}

La Loma de la Cruz es mucho más que una pintoresca elevación a la altura de una ventana, es un conjunto valores históricos, culturales y patrimoniales. Por tanto, este sitio constituye uno de los más promocionados en el territorio destacando fundamentalmente los siguientes elementos: escalinata, altar de la Cruz, peregrinación a la cima, mirador, culto religioso, tradiciones, festividad y costumbres. Por lo que la difusión del sitio por parte de medios locales e internacionales es notable, destacándose los siguientes:

- Periódico Ahora

- Periódico La Luz

- Publicaciones variadas de Ediciones Holguín, tales como:

- Holguín colonial: páginas de su historia, publicado en 1992.

- Holguín desde sus inicios hasta 1898, publicado en 1992.

- Imágenes holguineras, publicado en 1994.

- La Loma de la Cruz, publicado en 1995.

- Cálculo del Índice de atractividad de la "Loma de la Cruz"

Para ello se confecciona la Matriz de Índice de Atractividad (Ver Tabla 4) y se aplican las fórmulas 1 y 2.

Fórmula 1 Cálculo del Valor del Atributo: Va $=\operatorname{Im} \times \operatorname{Pr}$

Fórmula 2 Cálculo de Índice de Atractividad: $\sum\left(V a_{1}+V a_{2}+\cdots+V a_{30}\right) / N$

La Loma de la Cruz es un sitio que cuenta con atributos que han permitido que, con el paso del tiempo, se convirtiera en el símbolo insigne de la provincia, que desde cualquier parte de la ciudad de Holguín se, muestran los carteles y señalizaciones indicando como acceder a este hermoso lugar. Es un sitio que se caracteriza por su originalidad, exclusividad y valores estéticos-escénicos, por lo que capta la atención de todo visitante que se encuentre en los alrededores. Es un producto turístico con posibilidades para integrar conjuntos, multiproductos y multidestinos, de acuerdo a la diversificación de las ofertas en cuanto a la naturaleza, turismo rural, manifestaciones sociales y recreación.

Cuenta con un restaurante en su cima, que se distingue por la calificación y amabilidad del personal, así como la relación costo-calidad-precio. De forma general se caracteriza por satisfacer a todos los visitantes, pues aparte de la seguridad e higiene y la calidad ambiental que muestra, es el mirador natural de Holguín ofrece increíbles vistas, acompañada de tradiciones.

Por otro lado, existen atributos que frenan su desarrollo como producto turístico como es el caso del transporte, acceso y viabilidad, pues no existe ningún transporte que facilite el 
acceso a la cima, por lo que si el visitante quiere disfrutar de los atractivos que se encuentran en la cima tienen que acceder por los escalones o disponer de un medio de transporte propio. No cuenta con ninguna instalación de alojamiento y ha sufrido afectaciones por fenómenos naturales tales como huracanes e incendios, por lo que su estado de conservación, así como las condiciones del entorno se han ido deteriorando.

Cabe destacar que, como principal obstáculo, en cuanto a su desarrollo esta que la Loma de la Cruz no se gestiona correctamente como recurso turístico.

Lo antes expuesto se evidencia a continuación en la siguiente matriz de índice de atractividad. Donde al calcular el Valor del Atributo, aplicando la Fórmula 1, se obtuvo un resultado de 415, y luego aplicando la Fórmula 2, para calcular el Índice de Atractividad, se obtiene un aproximado de 18.83, lo que significa que la Loma de la Cruz es un sitio atractivo, y tiene un alto índice de atractividad.

Tabla 4. Matriz de Índice de Atractividad

\begin{tabular}{|c|c|c|c|c|c|c|c|c|c|c|c|}
\hline \multirow[t]{2}{*}{ No } & \multirow{2}{*}{\multicolumn{2}{|c|}{ ATRIBUTOS(a) }} & \multicolumn{3}{|c|}{ IMPORTANCIA(Im) } & \multicolumn{5}{|c|}{$\begin{array}{c}\text { POSICIÓN } \\
\text { RELATIVA(Pr) }\end{array}$} & \multirow{2}{*}{$\begin{array}{c}\text { VALOR } \\
\text { DEL } \\
\text { ATRIBUTO } \\
\text { (Va) }\end{array}$} \\
\hline & & & 1 & 2 & 3 & $\begin{array}{c}- \\
10\end{array}$ & $\begin{array}{l}- \\
5\end{array}$ & $\begin{array}{l}- \\
2\end{array}$ & 25 & 10 & \\
\hline 1 & $\begin{array}{l}\text { Señalización } \\
\text { información } \\
\text { turística } \\
\text { ambiental }\end{array}$ & $\begin{array}{l}\mathrm{e} \\
\mathrm{y}\end{array}$ & & & 3 & & & & & 10 & 30 \\
\hline 2 & $\begin{array}{l}\text { Transporte, acc } \\
\text { y vialidad }\end{array}$ & eso & & & 3 & & & - & & & -6 \\
\hline 3 & $\begin{array}{l}\text { Tiempos } \\
\text { desplazamiento }\end{array}$ & de & & 2 & & & & & 2 & & 4 \\
\hline 4 & Originalidad & & & & 3 & & & & & 10 & 30 \\
\hline 5 & Exclusividad & & & & 3 & & & & & 10 & 30 \\
\hline 6 & $\begin{array}{l}\text { Nivel } \\
\text { conservación } \\
\text { condiciones } \\
\text { entorno }\end{array}$ & $\begin{array}{r}\text { de } \\
\mathrm{y} \\
\text { del }\end{array}$ & & & 3 & & & - & & & -6 \\
\hline 7 & $\begin{array}{l}\text { Valores estéti } \\
\text { escénicos } \\
\text { (visuales) }\end{array}$ & os- & & & 3 & & & & & 10 & 30 \\
\hline 8 & Capacidad de ca & rga & 1 & & & & & & & 10 & 10 \\
\hline 9 & $\begin{array}{l}\text { Capacidad } \\
\text { alojamiento }\end{array}$ & de & 1 & & & $\begin{array}{c}- \\
10\end{array}$ & & & & & -10 \\
\hline 10 & $\begin{array}{l}\text { Capacidad } \\
\text { restauración }\end{array}$ & de & & & 3 & & & & 5 & & 15 \\
\hline 11 & $\begin{array}{l}\text { Capacidad } \\
\text { servicios } \\
\text { equipamientos } \\
\text { apoyo }\end{array}$ & $\begin{array}{r}\text { en } \\
y \\
\text { de }\end{array}$ & 1 & & & & & & 2 & & 2 \\
\hline
\end{tabular}


Tabla 4. Matriz de Índice de Atractividad (continuación)

\begin{tabular}{|c|c|c|c|c|c|c|c|c|c|c|}
\hline \multirow[t]{2}{*}{ No } & \multirow[t]{2}{*}{ ATRIBUTOS(a) } & \multicolumn{3}{|c|}{ IMPORTANCIA(Im) } & \multicolumn{5}{|c|}{$\begin{array}{c}\text { POSICIÓN } \\
\text { RELATIVA(Pr) }\end{array}$} & \multirow{2}{*}{$\begin{array}{c}\text { VALOR } \\
\text { DEL } \\
\text { ATRIBUTO } \\
\quad(\text { Va) }\end{array}$} \\
\hline & & 1 & 2 & 3 & - & $\begin{array}{l}- \\
5\end{array}$ & $\begin{array}{ll}- & 2 \\
2 & \end{array}$ & 25 & 10 & \\
\hline 12 & $\begin{array}{ll}\text { Nivel } & \text { de } \\
\text { explotación }\end{array}$ & & & 3 & & & & 2 & & 6 \\
\hline 13 & $\begin{array}{l}\text { Captación de } \\
\text { atención y duración } \\
\text { media }\end{array}$ & & & 3 & & & & & 10 & 30 \\
\hline 14 & $\begin{array}{l}\text { Monotonía } \\
\text { variedad }\end{array}$ & & & 3 & & & & 2 & & 6 \\
\hline 15 & $\begin{array}{lr}\text { Necesidad } & \text { de } \\
\text { desarrollo } & \text { de } \\
\text { nuevas actividades }\end{array}$ & & 2 & & & & & 2 & & 4 \\
\hline 16 & $\begin{array}{l}\text { Actividades } \\
\text { participativas } \\
\text { potenciales } \\
\text { realizar }\end{array}$ & & & 3 & & & & 2 & & 6 \\
\hline 17 & $\begin{array}{l}\text { Diversificación de } \\
\text { ofertas: naturaleza }\end{array}$ & & & 3 & & & & & 10 & 30 \\
\hline 18 & $\begin{array}{l}\text { Diversificación de } \\
\text { ofertas: deportes y } \\
\text { aventura }\end{array}$ & & 2 & & & & $\begin{array}{l}- \\
2\end{array}$ & & & -4 \\
\hline 19 & $\begin{array}{l}\text { Diversificación de } \\
\text { ofertas: turismo } \\
\text { rural }\end{array}$ & & 2 & & & & & & 10 & 20 \\
\hline 20 & $\begin{array}{l}\text { Diversificación de } \\
\text { ofertas: } \\
\text { manifestaciones } \\
\text { culturales }\end{array}$ & & & 3 & & & & & 10 & 30 \\
\hline 21 & $\begin{array}{l}\text { Diversificación de } \\
\text { ofertas: recreación }\end{array}$ & 1 & & & & & & 5 & & 5 \\
\hline 22 & $\begin{array}{l}\text { Posibilidades para } \\
\text { integrar conjuntos, } \\
\text { multiproductos y } \\
\text { multidestinos }\end{array}$ & & & 3 & & & & 5 & & 15 \\
\hline 23 & $\begin{array}{l}\text { Calificación del } \\
\text { personal }\end{array}$ & & & 3 & & & & & 10 & 30 \\
\hline 24 & Amabilidad & & & 3 & & & & & 10 & 30 \\
\hline 25 & $\begin{array}{l}\text { Seguridad } \\
\text { higiene }\end{array}$ & & & 3 & & & & 5 & & 15 \\
\hline 26 & Calidad ambiental & & & 3 & & & & 5 & & 15 \\
\hline 27 & $\begin{array}{l}\text { Relación costo- } \\
\text { precio-calidad }\end{array}$ & & & 3 & & & & & 10 & 30 \\
\hline 28 & $\begin{array}{l}\text { Satisfacción del } \\
\text { cliente }\end{array}$ & & & 3 & & & & & 10 & 30 \\
\hline
\end{tabular}


Tabla 4. Matriz de Índice de Atractividad (continuación)

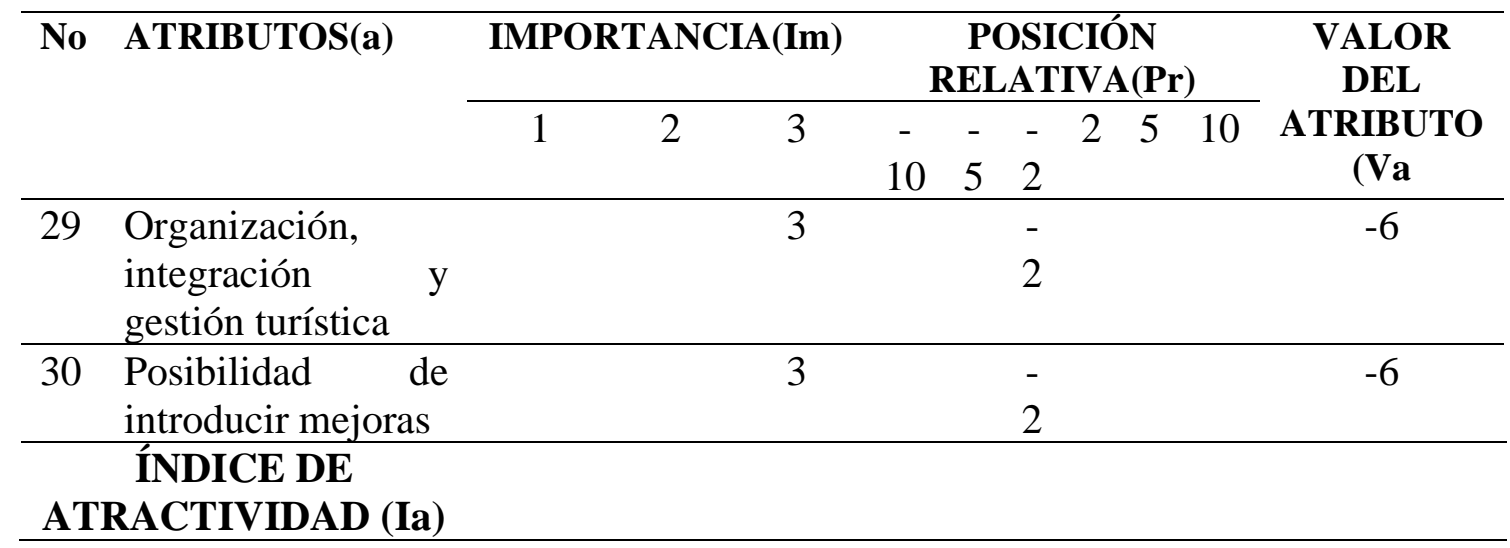

Fuente: Elaboración propia.

\section{Conclusiones.}

- La Loma de la Cruz es un sitio natural que encierra valores históricos y etnográficos que han hecho a través de los años que haya representado simbólicamente a la ciudad de Holguín.

- Existen problemas medioambientales asociados al incremento del turismo, el cual ha incidido negativamente en la conservación de elementos tanto naturales como patrimoniales, pues al incrementarse el número de visitas al sitio, también se produce un incremento de la acción del hombre sobre el espacio geográfico sobre el cual se localiza este producto turístico.

- En la actualidad se considera el mirador natural de la ciudad y un lugar significativo dentro de la celebración de las Romerías de Mayo, evento de gran connotación nacional e internacional. En su cima hay varias instalaciones, entre las que se destacan: la estación repetidora de señales de televisión, mirador, restaurante y plazoleta-mirador con torre de observación, sin embargo, no se gestiona correctamente como recurso turístico.

- De acuerdo a la evaluación cuantitativa y cualitativa del Índice de Atractividad se clasifica como un lugar atractivo y con un alto índice de atractividad.

\section{Referencias bibliográficas.}

Aranibar, V. H. (2021). Inventariación y seguimiento al desarrollo de los recursos turísticos. doi: https://doi.org/10.13140/RG.2.2.18944.97284

Beerli, A., \& Martin, J. D. (2004). Tourists' characteristics and the perceived image of tourist destinations: a quantitative analysis - a case study of Lanzarote, Spain. Tourism Management, 25, 623-636.

Beirman, D. (2003). Restoring Tourism Destinations in Crisis: A Strategic Marketing Approach. 
Cantú-Martínez, P. C. (2018). Desarrollo sustentable: cultura. patrimonio cultural y natural en México. Turismo y Sociedad, XXIII, pp. 25-40. doi: https://doi.org/10.18601/01207555.n23.01

Carvajal-Zambrano, G. V., \& Lemoine-Quintero, F. A. (2018). Análisis de los atractivos y recursos turísticos del cantón San Vicente. El Periplo Sustentable, No.34, 164 184.

Cepero, M. B., Hidalgo, D. M. G., Ramírez, N. E., Cruz, L. B., \& Beltrán, E. J. (2016). Análisis del producto turístico "Loma de la Cruz". (Trabajo de curso), UHO, Holguín.

Ciudad de Holguín - Ciudad de los Parques. (2018). Loma de la Cruz. from http://www.ciudaddeholguin.org/es/geografia/loma-de-la-cruz.html

Cubaism. (2018). Ciudad de Holguín - Ciudad de los Parques. Loma de la Cruz. from http://www.ciudaddeholguin.org/es/geografia/loma-de-la-cruz.html

Díaz, R. (2013). Propuesta de un Parque Temático en los Planos Inclinados de Pinares de Mayarí. (Trabajo de Diploma en opción al título de Licenciatura en Estudios Socioculturales), Instituto Superior Minero-Metalúrgico Dr. Antonio Nuñéz Jiménez Moa.

Gambarota, D. M., \& Lorda, M. A. (2018). Análisis de los atractivos turísticos de una ciudad intermedia: El caso de Bahía Blanca, Argentina. Revista Interamericana de Ambiente y Turismo, 14, $N^{o}$ 2, 135-160.

González, W. B., Oca, Z. G. M. d., \& González, R. B. (2010). Diccionario Geográfico de Holguín.

Guzmán-Vilar, L., \& García-Vidal, G. (2014). "La gestión turística del patrimonio cultural en Holguín, Cuba. Retos para una investigación futura". Revista Caribeña de Ciencias Sociales.

Igarza, L. M. Z., Milanés, O. G., Gómez, J. L. R., Angulo, M. M. R., Ramírez, A. D., Peña, D. B., . . Pioto, V. A. T. (2008). Perspectivas del Medio Ambiente Urbano: GEO Holguín. La Habana: Academia.

Isla Local. (2017). La Loma de la Cruz: símbolo por excelencia de Holguín. from https://www.islalocal.com/la-loma-la-cruz-simbolo-excelencia-holguin/

Lee, C., Ou, W., \& Huang, H. (2009). A study of destination attractiveness through domestic visitors' perspectives: the case of Twain's hot spring tourism sector. Asia Pacific Journal of Tourism Research, 14(1), 17-38.

Magallanes, A. J. (2004). Atractividad de mercados turísticos. México: Internacional Thomson Editores. 
Medina-Labrada, J., Pérez-Ricardo, E. d. C., \& Riquenes-Gainza, M. d. 1. M. (2019). Atractividad - Competitividad de destino turístico. Evaluación de Holguín para el mercado estadounidense. Retos Turísticos, Vol. 18, No. 1.

Mondéjar, J. J., \& Vargas, M. (2009). Construción de un modelo para el análisis motivaciones sobre la elección de un destino turístico. Estudios y Perspectivas en Turismo, 18, $400-413$.

Obregón, Á. P. (1995). La Loma de la Cruz. La Habana: Editorial Pablo de la Torriente.

Obregón, Á. P. (2010). La Ciudad de los Parques. Holguín: Ediciones Holgín.

Obregón, Á. P. (2015). Juventud Rebelde. Un cerro en el corazón. Héctor Carballo Hecheverría. from http://www.juventudrebelde.cu/cuba/2015-09-17/un-cerro-enel-corazon

OMT. (2019). Panorama del turismo internacional (Edición 2019 ed.). Madrid: UNWTO.

Ortiz-Liñán, M. E., \& Vázquez-Solís, V. (2021). El inventario turístico: un instrumento de gestión territorial sustentable del turismo en atractivos naturales del estado de San Luis Potosí, México. Investigaciones Turísticas (21), pp. 305-327. doi: https://doi.org/10.14198/INTURI2021.21.14

Pupo, L. E., Suárez, J. c. J., Pérez, R. L., \& Figueroa, D. L. (2012). Trabajo de curso de las asignaturas Comercialización Turística, Panorama de la Cultura Cubana y Geografía Turística y Medio Ambiente. (Trabajo de curso), UHO.

Radio Rebelde. (2015). Desde la Loma de la Cruz, el Papa Francisco bendice a los holguineros. from http://www.radiorebelde.cu/noticia/desde-loma-cruz-papafrancisco-bendice-holguineros-20150921/

$\begin{array}{lllll}\text { Rivera, } & \text { S. } & \text { R. } & \text { (2008). } & \text { Blogspot. }\end{array}$ http://estudiosdelturismo.blogspot.com/2009/05/conceptos-de-patrimonioatractivo.htm

UNESCO. (2017). Organización de las Naciones Unidas para la Educación, la Ciencia y la Cultura. Patrimonio. Indicadores Unesco de Cultura para el desarrollo. Manual metodológico. Francia.

Wu, T.-C., Xie, P. F., \& Tsai, M.-C. (2015). Perceptions of attractiveness for salt heritage tourism: A tourist perspective. Tourism Management, 51(3), 201-209.

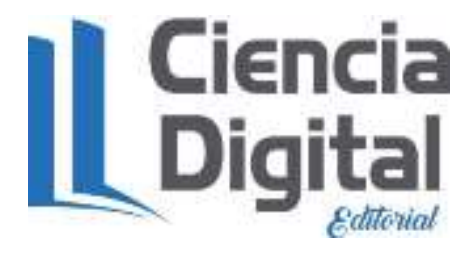




\section{PARA CITAR EL ARTÍCULO INDEXADO.}

Pérez-Labrada, S., Díaz-Pompa, F., Serrano-Leyva, B., \& Cruz-Aguilera, N. (2021). Análisis de recursos turísticos para el cálculo del índice de atractividad. Caso de estudio Loma de la cruz, Holguín. AlfaPublicaciones, 3(3.2), 51-68. https://doi.org/10.33262/ap.v3i3.2.97

\section{\Ciencia}

El artículo que se publica es de exclusiva responsabilidad de los autores y no necesariamente reflejan el pensamiento de la Revista Alfa Publicaciones.

El artículo queda en propiedad de la revista y, por tanto, su publicación parcial y/o total en otro medio tiene que ser autorizado por el director de la Revista Alfa Publicaciones.
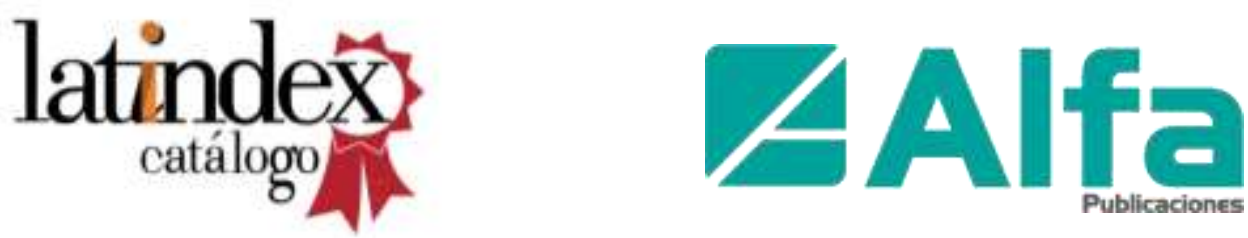